REVISTA CIENCIAS BIOMÉDICAS

PRESENTACIÓN DE CASOS CLÍNICOS

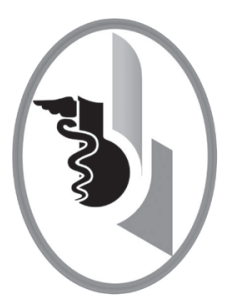

\title{
HIDROTÓRAX FETAL ASOCIADO A HIDROPESIA ¿MANEJO EXPECTANTE O INTERVENCIONISTA?
}

\author{
FETAL HYDROTHORAX ASSOCIATED TO HYDROPS: \\ EXPECTANT OR INTERVENTIONIST MANAGEMENT?
}

\author{
Ortiz Escobar Alexa Gianina ${ }^{1}$ \\ Meneses Ruiz Katherine ${ }^{2}$
}

Correspondencia: alexagianina05@hotmail.com

Recibido para evaluación: Noviembre - 18-2011. Aceptado para publicación: enero - 31- 2011.

\section{RESUMEN}

El hidrotórax fetal es la acumulación de líquido libre en la cavidad pleural fetal. Se asocia muy frecuentemente al hidrops fetalis entidad de elevada mortalidad perinatal, de etiología multifactorial y caracterizado por anasarca generalizada, con acumulación de líquido en algunas cavidades serosas del organismo fetal. Es importante establecer con antelación esta condición, ya que debe conocerse la causa más probable, para ayudar en la mejor reanimación al nacimiento. Se presenta el reporte de un caso en la ciudad de Cartagena. Colombia. Paciente de 35 años de edad, secundigestante de grupo sanguíneo RH positivo, con antecedente quirúrgico de resección de tumor dermoide en pared abdominal, músculos rectos abdominales, con colocación de malla de propileno. Embarazo anterior sin alteraciones. A las treinta y una semana de gestación se hace diagnóstico ecográfico de derrame pleural bilateral fetal. Se realiza intervención intrauterina para drenar líquido y colocación de dren pleuroamniotico. Por no presentar mejoría se decide nuevamente toracocentesis fetal. Tampoco se presenta mejoría, por lo que se interrumpe embarazo por medio de cesárea. El producto al nacer presenta signos de insuficiencia respiratoria y se recurre a asistencia ventilatoria mecánica con altos parámetros. Persistió el derrame pleural por lo que se realizó drenaje y colocación de tubo de tórax, con evolución poco favorable y muerte neonatal. Actualmente no existe consenso mundial del manejo del hidrotórax fetal asociado o no a hidropesía, se plantean factores a tener en cuenta que guiaran a la decisión de un manejo expectante o intervencionista, basándose en la causa desencadenante. Rev.cienc.biomed. 2010; 2 (1): 121-129

\section{PALABRAS CLAVES}

Hidrotórax fetal. Hidrops fetalis. Derivación pleuroamniotica.

\section{SUMMARY}

Background: Fetal hydrothorax is the accumulation of free fluid in pleural cavity of fetus. It is very often associated to hydrops fetalis which is a serious entity with a high perinatal mortality, multifactorial etiology and it is characterized by generalized anasarca, with accumulation of fluid in some serous cavities of the fetus. It is important to know beforehand if this condition is present in order to establish the most probable origin and to be prepared to administer optimal reanimation management of the neonate at birth. The care given to a newborn with hydrops fetalis is always a challenge for the neonatologist. We present a report of a case in the city of Cartagena. Case report:

\footnotetext{
1 Médico, Universidad del Sinú, seccional Cartagena. Médico de Planta Clínica Universitaria San Juan de Dios. Cartagena. Colombia.

2 Médico, Universidad del Sinú, seccional Cartagena. Médico de Planta del Hospital Naval de Cartagena. Colombia.
} 
We present the case of a pregnant woman, O positive blood type, with important preceding surgery, without alterations in previous pregnancy. Her fetus in 31st week was diagnosed with bilateral pleural effusion. The especialist performed intrauterine intervention to drain fluid twice without improvement, therefore pregnancy was interrupted through cesarean section. The newborn presents signs of respiratory failure requiring mechanical ventilatory assistance with high parameters. The pleural effusion persisted, so drainage and insert of thoracic tube was made obtaining an unfavorable evolution with neonatal death. Conclusions: Currently, there's not a world consensus of the management of fetal hydrothorax associated or not to hydrops; we present highlights to guide the decision of an expectant or interventionist management, based on underlying cause. Rev.cienc.biomed. $2011 ; 2$ (1): 121-129

\section{KEY WORDS}

Hydrops fetalis. Congenital anomalies. Non-immune hydrops fetalis.

\section{INTRODUCCIÓN}

El hidrotórax fetal es la acumulación de líquido libre en la cavidad pleural fetal, aunque esta condición se detecta frecuentemente en el contexto de hidrops fetalis, el hidrotórax fetal primario es una patología rara, estimándose su incidencia en 1 de cada 15.000 embarazos (1).

Es considerada una patología grave, con una tasa de mortalidad perinatal cercana al $50 \%$ (2), concentrándose los casos de mal pronóstico en aquellos asociados a hidrops fetal no inmune, lo que destaca la importancia del diagnóstico prenatal oportuno y el manejo subsecuente. No existe consenso en la literatura en relación al manejo óptimo de esta patología, considerándose opciones que incluyen desde el aborto terapéutico en países en que este está legalizado, observación seriada ultrasonográfica y terapia fetal. En este último caso, las intervenciones incluyen toracocentesis fetal e inserción de shunt pleuroamniótico $(1,2)$.

El diagnóstico antenatal de hidrotórax fetal fue comunicado por primera vez en 1977 por Potter (2), quien reconoció que esta entidad, denominada desde entonces hidrops fetalis no inmune, no representaba una enfermedad específica, sino más bien una manifestación tardía de muchas enfermedades severas. Cuando Potter describió por primera vez el hidrops fetalis no inmune, esta entidad constituía menos del $20 \%$ de los casos de hidrops; en cambio, desde el advenimiento de profilaxis efectiva contra la sensibilización $\mathrm{Rh}(\mathrm{D})$ ha disminuido la incidencia del hidrops inmune, la frecuencia relativa del hidrops fetalis no inmune ha aumentado al $90 \%$ (2).
En las últimas décadas, la amplia difusión del ultrasonido en el cuidado prenatal y la mejoría de la resolución de imágenes ha permitido realizar el diagnóstico prenatal de hidrotórax en forma más frecuente y en etapas más precoces, siendo posible realizar el diagnóstico en el primer trimestre (3). La mayoría de los casos, sin embargo, son detectados en la segunda mitad del embarazo, fecha en la cual el diagnóstico es relativamente fácil.

El hidrops fetal, también conocido como hidropesía fetal, es una condición clínica que se caracteriza por la acumulación anormal de líquido que lleva a un exceso extracelular en los tejidos blandos (edema) y en algunas de las cavidades serosas del feto, derrame pericárdico, derrame pleural, ascitis (3). La principal clasificación del hidrops se basa en dos grupos de etiologías: inmune y no inmune (3).

En el caso del hidrops fetal inmune (10\%), este puede desarrollarse por la incompatibilidad del sistema Rh entre la madre y el feto. Cuando una madre que es factor Rh negativo tiene un feto que es factor Rh positivo, el sistema inmune desarrolla anticuerpos contra los eritrocitos del feto, provocándole anemia (6). Luego, debido a que los órganos del feto no son capaces de compensar la anemia, se puede desarrollar la hidropesía (3). El corazón comienza a desarrollar insuficiencia y grandes cantidades de líquido se acumulan en los tejidos y órganos fetales pudiendo ocasionar finalmente la muerte fetal (4).

El hidrops no inmune presenta una incidencia del $90 \%$ (3), se define por la ausencia 
materna de anticuerpos circulantes dirigidos a antígenos eritrocitarios e incluye todas las demás enfermedades o complicaciones que pueden afectar la capacidad del feto para controlar los líquidos; no existe un mecanismo único que explique la hidropesía no inmune. Dentro de sus causas más frecuentes se encuentran las cardiopatías (25$30 \%$ ), malformaciones o síndromes genéticos $(22 \%)$, anomalías cromosómicas $(16 \%)$, infecciones $(5 \%)$, alteraciones hematológicas $(5 \%)$, metabolopatías $(2 \%)$ e idiopáticas (22\%) (6).

\begin{tabular}{|l|}
\multicolumn{1}{|c|}{ TABLA NO $\mathbf{1}$} \\
CAUSA DE HIDROPESÍA FETAL \\
\hline ANEMIA CRONICA GRAVE IN UTERO \\
Parvovirus B19 - 20\% \\
Alfatalasemia homocigota \\
Transfusión feto-materna o gemelo-gemelo \\
Deficiência de glucosa 6 fosfato \\
deshidrogenasa \\
Anemia fetal de Diamon Blackfan \\
Leucemia congénita \\
\hline FALLA CARDIACA \\
Cardiomiopatía o miocarditis grave \\
Cierre prematuro del foramen oval \\
Malformación arteriovenosa \\
Arritmias intrauterinas \\
\hline HIPOPROTEINEMIA \\
\hline ENFERMEDAD RENAL \\
Nefrosis congénita \\
Trombosis de la vena renal \\
\hline HEPATITIS CONGÉNITA \\
\hline INFECCION INTRAUTERINA \\
Sífilis \\
Toxoplasmosis \\
Citomegalovirus \\
Parvovirus B19 \\
\hline MISCELANEAS \\
Diabetes Mellitus \\
Embarazo múltiple \\
Hipertensión inducida por el embarazo \\
Trombosis umbilical o de la vena coriónica \\
Neuroblastoma fetal \\
Malformación pulmonar adenomatoidea \\
quística \\
Linfangiectasia pulmonar \\
Corioangioma de la placenta \\
Leucemia transitoria en pacientes con \\
síndrome de down \\
Anemia carencial \\
\hline CROMOSOPATIAS \\
Trisomías 13, 18 y 21 \\
Monosomía XO \\
Mosaicismo \\
\hline
\end{tabular}

La frecuencia de aparición del hidrops no inmune varía según series de entre $1 / 1500$ y $1 / 4000$ partos y el pronóstico es malo, con una mortalidad global muy alta cercana al $90 \%$, a pesar de que cada vez disponemos de más medios para un diagnóstico cuidadoso y podemos seleccionar los casos susceptibles de tratamiento intraútero (3). En caso contrario, es importante precisar el diagnóstico para ofrecer un consejo prenatal de cara a futuras gestaciones, aún así, uno de cada cuatro casos quedan sin diagnosticar.

La atención de un neonato con hidrops fetal es un reto. Cuando se establece esta condición, se debe tratar de conocer la causa más probable que la originó para ayudar en la mejor reanimación al nacimiento; por lo que cabe resaltar la importancia de realizar un buen diagnóstico prenatal (4).

Se debe realizar una historia clínica detallada incluyendo antecedentes personales como: la raza (personas del sureste asiático es mas frecuente que presenten alfa-talasemia en homocigotos), patologías como LES, diabetes ó pérdidas de anteriores embarazos que podrían estar relacionadas con errores del metabolismo o con anomalías cromosómicas (4). La infección por parvovirus B19 que es más frecuente en docentes, cuidadores de guarderías y en personas en contacto doméstico con niños pequeños; La analítica materna, que incluirá hemograma completo, grupo sanguíneo y Rh, Coombs indirecto e investigación de otros anticuerpos irregulares, serologías: IgG e IgM de parvovirus B19, toxoplasmosis, rubéola, sífilis y citomegalovirus, herpes, entre otros, En casos seleccionados descartar diabetes y, si se sospecha anemia no filiada, electroforesis de hemoglobinas e investigación de déficit de G6PD. Ecografía en búsqueda de cardiopatías y marcadores ecográficos de infección como microcefalia, ventriculomegalia y calcificaciones intracraneales ó hepáticas (4).

Alteraciones metabólicas pueden presentarse cuando el hidrops se asocie a restricción en el crecimiento intrauterino, miocardiopatía hipertrófica, hipomotilidad o acinesia, anomalía esquelética o hepatoesplenomegalia. El examen ecográfico in- 
cluirá estudio Doppler para valorar el estado fetal (3) y la velocidad máxima de la arteria cerebral media que presenta alto valor predictivo de anemia fetal. La ecocardiografía es útil para descartar anomalías estructurales (más frecuentemente si presentan afectación valvular) o arritmias; se pueden realizar otros procedimientos invasivos, como la cordocentesis, La amniocentesis, y biopsia de corion para estudios adicionales. En los casos de muerte fetal o neonatal y de interrupción legal de la gestación es muy importante la realización de necropsia que ayudará a aclarar la etiología hasta en un $92 \%$ de los casos (7). Sin esta prueba no se puede concluir el estudio del Hidrops no inmune.

\section{CASO CLÍNICO}

Paciente femenina de 35 años de edad en su segunda gestación, con los siguientes antecedentes quirúrgicos de importancia: Hace cuatro años resección de tumor dermoide en pared abdominal más resección de músculos rectos anteriores del lado derecho con colocación de malla de propileno. Embarazo anterior: gestación sin alteraciones, parto por cesárea a la semana 37 sin complicaciones. Estudios prenatales: glicemia, VIH, toxoplasma (Ig G e Ig M), citomegalovirus ( Ig $G$ e Ig $M$ ), rubéola y herpes tipo 1 y 2: negativos. Ecografía pélvica transvaginal reporta: Embarazo de 7 semanas + 4 días + embrión único vivo + miomatosis uterina (múltiples miomas intramurales; los de mayor tamaño $(28 \mathrm{~mm}$., $35 \mathrm{~mm}$., $17 \mathrm{~mm}$. y $16 \mathrm{~mm}$.) ubicados en pared anterior del cuerpo uterino.

Continuó control prenatal sin alteraciones, se realizó nueva ecografía en el termino de 1 mes y reportó embarazo de 12 semanas, miomatosis uterina (región fúndica subserosa mioma de $3 \mathrm{~cm}$. y en región intramural de igual tamaño), placenta previa oclusiva total, translucencia nucal: $1.2 \mathrm{~mm}$. , hueso nasal presente y ductus venoso con patrón espectral normal. (Figura No 1, Figura No 2, Figura No 3).

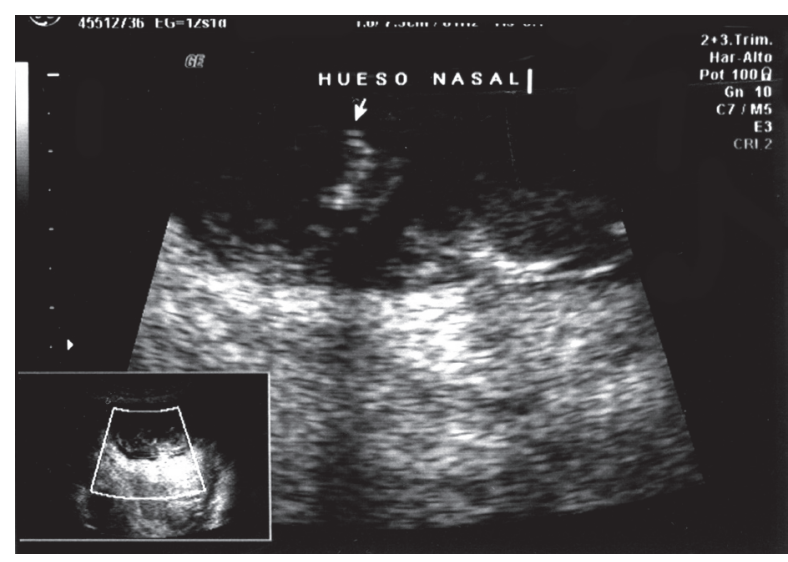

Figura No 1.

Presencia de hueso nasal fetal. Edad gestacional 12 semanas

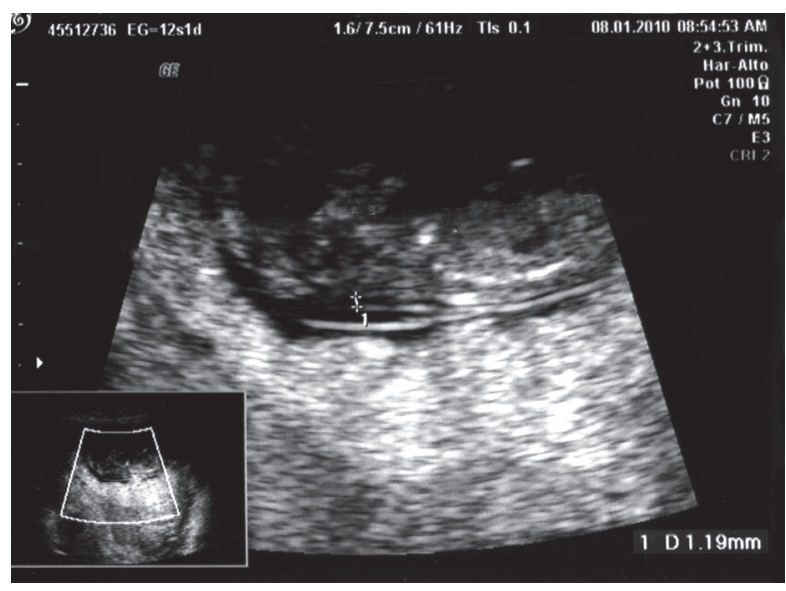

Figura No 2.

Translucencia nucal fetal: 1.2 milímetros. Edad gestacional 12 semanas

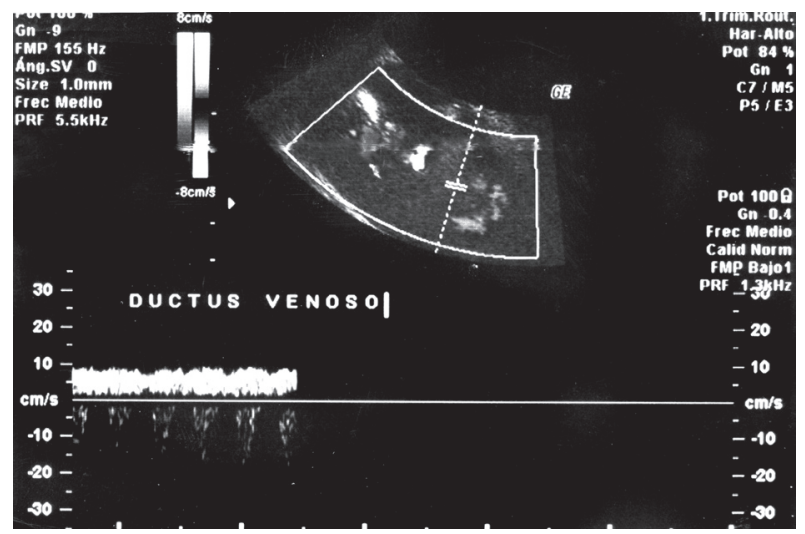

Figura No 3.

Ductus venoso fetal con patrón espectral normal. Edad gestacional 12 semanas 
Un mes después presentó episodio de amenaza de aborto siendo manejada con reposo, progesterona oral e intravaginal. Se realizó ecografía la cual reportó embarazo de 16 semanas más feto único vivo, placenta previa oclusiva total, útero con 3 miomas (pared anterior de $29 \mathrm{~mm}$. , pared lateral derecha $32 \mathrm{~mm}$., pared posterior $41 \mathrm{~mm}$.). Días después presentó episodio de síndrome gripal, con accesos de tos acentuados, que fue manejado con jarabe antitusivo y mebucaína, con mejoría en una semana.

Ecografía tridimensional a la semana 19 de gestación: feto único transverso sin alteraciones, placenta lateral izquierda grado 0, índice de líquido amniótico normal, cuatro miomas uterinos, uno de $58 \times 41$ centímetros, intramural ístmico izquierdo que hace contacto con la placenta. Otra ecografía tridimensional dos semanas después reportó hallazgos similares.

Experimentó nuevo episodio de sangrado escaso, manejada con progesterona. Al realizar paraclínicos correspondientes al segundo trimestre de embarazo, todos fueron reportados dentro de los límites normales. Nueva ecografía doppler color obstétrico: embarazo de 31 semanas + feto único vivo, presencia de derrame pleural derecho con colapso de ese pulmón. No hay signos de sufrimiento fetal. Peso fetal estimado en 1600 más o menos 230 gramos. Índice de líquido amniótico: 12 (normal), placenta lateral izquierda grado I, no previa, no se observan alteraciones en el sistema nervioso fetal. Los riñones son de aspecto normal, vejiga llena sin defectos, la columna vertebral se visualiza de aspecto normal, no fue posible visualizar adecuadamente el corazón, ya que el dorso estaba anterior. Se realizó Doppler color del cordón y de la arteria cerebral media observándose un índice de pulsatilidad de 0.8 y 2.0 lo cual es normal. Frecuencia cardiaca fetal de 130-184 por minuto con los movimientos del feto. El Doppler del ductus venoso y la aorta fetal también fueron normales.

Con el diagnóstico de hidrotórax fetal se decidió realizar maduración pulmonar y nuevo control ecográfico en 5 días. Se realizó control imagenológico y se observó acumulación de líquido en ambos espacios pleurales con predominio de hidrotórax derecho, pulmones en alas de murciélago por compresión, el derecho ocupa aproximadamente el $30 \%$ del hemitórax y el izquierdo el 50\%. Figura No 4. Corazón con eje de $45^{\circ}$ con cuatro cámaras, tabique interventricular íntegro, tractos de salida en posición anatómica normal. Resto del estudio normal.

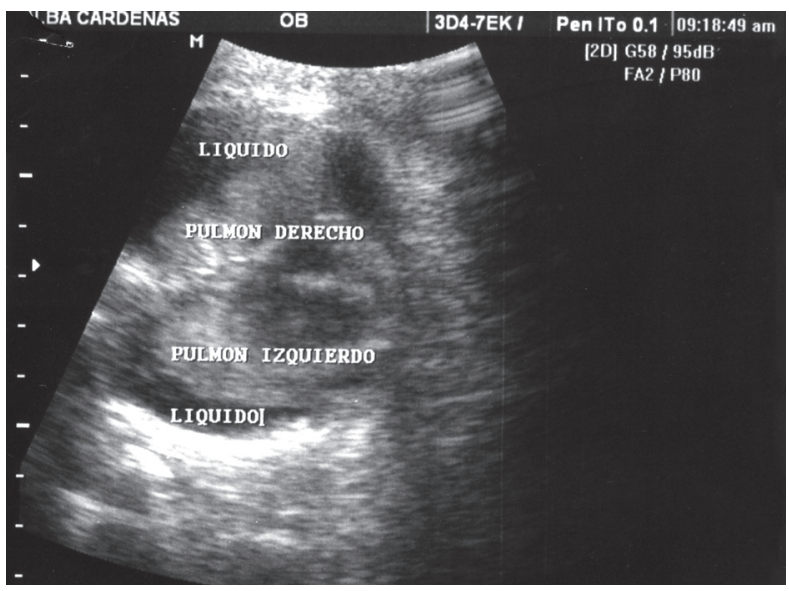

Figura N04.

Edad gestacional 31 semanas. Acumulación de líquido en ambos espacios pleurales. Pulmones en ala de murciélagos por compresión. El pulmón derecho ocupa el $30 \%$ del hemitórax, mientras que el pulmón izquierdo ocupa el $50 \%$.

Nuevos estudios de VIH, toxoplasma, citomegalovirus, herpes 1 , herpes 2, rubéola y hepatitis. Son informados como normales. Se ordena hospitalización. Se solicitó perfil toxémico, proteinuria en 24 horas, urianálisis, urocultivo, ecografía Doppler obstétrica, cervicometría, ecocardiograma, anticuerpos anticardiolipinas, anticoagulante lúpico y anticuerpos antinucleares

Resultados de algunos paraclínicos: Hemocultivos negativos, hemoclasificación $\mathrm{O}$ (+), calcio: $9.2 \mathrm{mg} / \mathrm{dL}$, creatinina: $1.4 \mathrm{mg} /$ $\mathrm{dL}$, glucosa: $123 \mathrm{mg} / \mathrm{dL}$, BUN: $28.5 \mathrm{mg} /$ $\mathrm{dL}$, potasio: $7 \mathrm{mg} / \mathrm{dL}$, sodio: $1.24 \mathrm{mg} / \mathrm{dL}$, TSH negativa, VDRL no reactivo, estudio de liquido pleural pleura derecha: glucosa 71 $\mathrm{mg} / \mathrm{dL}$, proteínas $2.5 \mathrm{mg} / \mathrm{dL}$, bacterias: no, polimorfos: no, hematíes: 15-20xc, LDH: 234U/L, colesterol: $68 \mathrm{U} / \mathrm{L}$. Líquido de pleura izquierda: glucosa: $65 \mathrm{mg} / \mathrm{dL}$, proteínas: $2.4 \mathrm{mg} / \mathrm{dL}$, bacterias: no, polimorfos: no, hematíes: 10-12xc, LDH:224U/L, cultivos negativos a las 72 horas de incubación. 
Previo consentimiento informado se realizó toracocentesis más derivación toracoamniótica bilateral. Se informó a la paciente que interviniendo, la tasa de mortalidad de la patología es cercana al 60\%, comparada con la conducta de no intervención la cual es del $100 \%$. Se solicita Test de Fisher para cromosoma 13, 18, 21 y sexuales.

Se realizó drenaje de líquido pleural bilateral, 100 cc. del hemitórax derecho y 90 cc. del izquierdo. Bajo guía ecográfica se realizó derivación toracoamniótica derecha con fácil colocación de catéter de Harrison. Fue fallido el intento de colocación del catéter del lado izquierdo. Al finalizar el procedimiento se observó re-expansión pulmonar derecha completa, persistencia de hidrotórax izquierdo del 50\% y movimientos respiratorios. Frecuencia cardiaca fetal 140 por minuto. Ausencia de ascitis.

A los dos días de haber realizado la intervención fetal, la ecografía mostró de nuevo hidrotórax bilateral. Se decidió manejo expectante y control ultrasonográfico al día siguiente; el nuevo estudio demostró persistencia del derrame pleural y además se evidencia ascitis leve. Nueva toracocentesis fetal bilateral, fue realizada sin dificultades. Hay evolución favorable y ausencia de actividad uterina. Se decide control ecográfico a los dos días del procedimiento y perfil biofísico fetal. Resultado de este ultimo: normal (8/8), mientras en la ecografía se observó evolución poco satisfactoria del derrame pleural bilateral. Se decidió realizar cesárea.

Se obtuvo producto con Apgar al minuto de 5 y de 8 a los cinco minutos. Inmediatamente el cirujano pediatra realizó toracocentesis bilateral, aspira $100 \mathrm{cc}$. de líquido cetrino espeso del lado derecho y $80 \mathrm{cc}$ del lado izquierdo. El neonato presentó Ilanto espontáneo pero con cianosis generalizada y tiraje universal, requiriendo intubación orotraqueal y asistencia ventilatoria con Fio2 del $100 \%$ y presión inspiración de 20, mejorando rápidamente hasta saturación del $100 \%$, cardiopulmonar normal, abdomen sin visceromegalias, diuresis negativa. La radiografía de tórax mostró reexpansión pulmonar bilateral y mínimo derrame pleural derecho que no ameritó colocación de tubo de tórax o nueva toracocentesis. Se ordenó traslado a UCI neonatal.

Al día siguiente el control radiográfico señaló infiltrado bilateral compatible con membrana hialina por lo que se decidió administrar surfactante exógeno. Evolución del neonato a la hora, evidencia estado crítico, en ARM (Asistencia respiratoria mecánica) con parámetros elevados, con mala saturación al intento de disminuir Fio2 y PIM (presión inspiratoria máxima). Pronóstico reservado. Se aumenta Fio2 al 100\% mejorando la saturación. Nueva radiografía de tórax muestra imagen compatible con hidrotórax bilateral. Gases arteriales indican acidosis respiratoria. Se realizó toracostomía cerrada de lado izquierdo puesto que el derrame es marcado en este hemitórax, se coloca tubo de tórax y se drenó aproximadamente 100 cc. de líquido pleural.

Al día siguiente se decidió cambiar tubo orotraqueal por continuar con pobre saturación y cianosis generalizada bajo ventilación mecánica con altos parámetros ventilatorios. Posterior al procedimiento se mejoró la perfusión, saturación, lo que permite disminuir los parámetros. Se observó hidrocele bilateral, presencia de anuria, por lo que se inicia furosemida y restricción hídrica. Radiografía de tórax evidenció aumento del derrame pleural derecho por lo que cirujano pediatra decide colocar tubo de tórax del lado derecho aproximadamente $100 \mathrm{cc}$. de líquido pleural cetrino amarillento. Figura No 5.

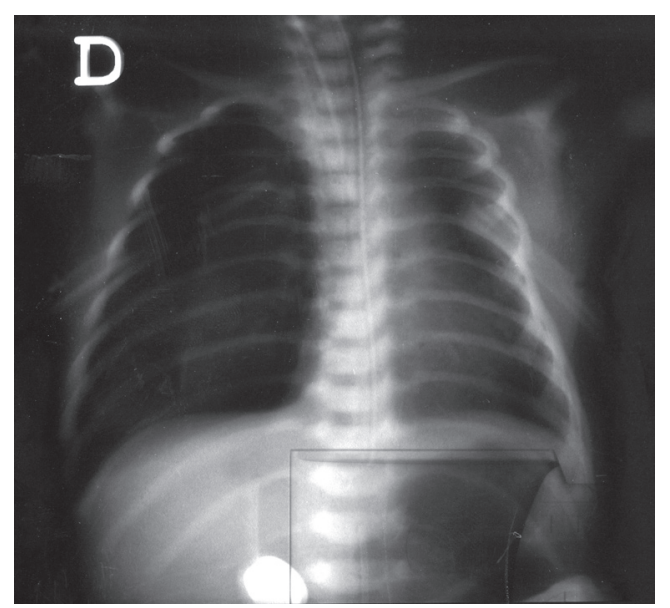

Figura No 5.

Tercer día de nacido. Tubo de tórax bilateral. Compresión pulmonar e hidrotórax bilateral. 
Al día siguiente el estado del recién nacido continúa critico. Malas condiciones generales, bajo ventilación mecánica, con parámetros 25/5/0, 5/0.3/40/100\% saturación: 79\%. Se aumentó PIM (presión inspiratoria máxima) y PEM (presión espiratoria máxima) mejorando saturación al $82 \%$. Persiste la anuria. Gases arteriales evidencian acidosis respiratoria severa más hipoxemia con acidosis metabólica severa, por lo que se aumentan parámetros y se indica corrección con bicarbonato. A la hora el paciente, presenta episodio de paro cardiorrespiratorio. No hay respuesta y el neonato fallece. Los padres no autorizan realizar necropsia por razones personales. A la semana siguiente se recibió reporte de cariotipo fetal en líquido amniótico negativo para alteraciones cromosómicas en cromosomas 13,18 y 21.

\section{DISCUSIÓN}

Casos de hidrotórax fetal asociado o no a hidropesía, son determinados con un estudio imagenológico de fácil acceso como la ultrasonografia (2), que hace parte del control prenatal en los tres trimestres de la gestación. Se realiza el diagnóstico precoz de esta entidad, ante la presencia de los siguiente signos ecográficos: fluido anecogénico en cavidad pleural fetal, compresión pulmonar, mediastino desplazado, corazón pequeño o normal, diafragma evertido. En la mayoría de los casos se acompaña de polihidramnios (1). Con el diagnóstico de hidrotórax fetal asociado o no a hidropesía se debe investigar la causa, siempre orientándonos a la clasificación de este, inmunológico o no inmunológico. Al definir un hidrops no inmunológico la conducta a seguir es descartar una cromosomopatía para así determinar la viabilidad del feto y precisar el manejo (1).

Al determinarse la causa se establece el manejo, ya sea expectante o intrevencionista. En el hidrops fetal inmune está indicado realizar transfusiones intrauterinas, para evitar la anemia fetal y posteriormente la acumulación de líquido en las cavidades $(1,2)$.

Se debe tener en cuenta que en un alto porcentaje de casos, aproximadamente en $22 \%$ no es posible identificar la causa, lo que hace difícil la decisión de realizar intervención o no (2). A favor de la conducta expectante está la alta mortalidad que tiene la patología, (50 a 96\%) con un promedio de un $76 \%$, la variabilidad de este porcentaje guarda relación con la causa, y la presencia de algunos factores pronóstico (1). Se han documentado tres factores de mal pronóstico: parto antes de la semana 32 de gestación, hidropesía y ausencia de intervención fetal $(1,2,5)$. La presencia de un factor pronóstico se asocia a mortalidad del $36 \%$, dos factores $77.8 \%$, si se presentan los tres factores la mortalidad asciende a un $97 \%$ (1). Se considera conducta expectante cuando aparece hidrotórax después de la semana 32 de embarazo, haciendo seguimiento ecográfico seriado, si no hay progresión del hidrotórax fetal y no aparece hidropesía $(1,2)$.

En cuanto al manejo intervencionista sus objetivos principales, teniendo en cuenta los principios de beneficiencia son salvar la vida o disminuir significativamente enfermedad irreversible en el feto, involucrar menos riesgos de muerte o morbilidad fetal $\mathrm{y} / \mathrm{o}$ materna; en el caso de hidrotórax fetal aislado o asociado a hidropesía se busca prevenir compresión pulmonar, la hipoplasia pulmonar, revertir los cambios hidrópicos y mejorar la función respiratoria postanatal. El procedimiento intervencionista consiste en realizar toracocentesis $y / o$ derivación pleuroamniótica; en el caso de la toracocentesis, tiene alta tasa de recidiva, sus ventajas es que este procedimiento mejora la sobrevida al realizarse anteparto en un $50 \%$ (2), mejora atención neonatal, necesidad de ventilación mecánica y reduce la necesidad de colocación de tubo de drenaje (2). La derivación pleuromaniótica consiste en un procedimiento invasivo transabdominal donde se inserta un catéter intrauterino bajo guía ecográfica hasta llegar al tórax fetal, posteriormente colocando un catéter de drenaje permanente en la cavidad pleural con comunicación al liquido amniótico, descomprimiendo el tórax y permitiendo así la reexpansión pulmonar. Las complicaciones de la derivación pleuroamniótica a nivel fetal que se han presentado son: torsión del cordón umbilical, brida amniótica, hipoproteinemia 
severa, hemomotórax; y en la madre: edema pulmonar, preeclampsia, trabajo de parto pretermino (1).

Son criterios para definir conducta: Edad gestacional y presencia aneuploidia $(1,2)$. Se justifica intentar intervenir antes de la semana 32 para tratar de dejar avanzar en edad gestacional, intentar la potencialidad de revertir el hidrops y mejorar la condición de recepción neonatal (1).

Cuando persiste el hidrotórax, aumente e inclusive aparezca hidropesía, a pesar de la primera intervención, la evidencia demuestra mejores resultados neonatales al no volver a realizar procedimiento de drenaje $\mathrm{y} / \mathrm{o}$ colocación de dren pleuroamniótico, mas aun cuando se ha llegado a la semana 32 de gestación puesto que se considera que una nueva intervención aumenta las riesgos fetales sobre las oportunidades neonatales; solo se considera realizar toracocentesis, antes de la cesárea seguida de ventilación mecánica de alta frecuencia en el nacimiento, buscando reclutamiento alveolar y evitar hipoplasia, asociado, drenaje neonatal y surfactante (1).

También en la medicina materno fetal, se debe hacer una evaluación y terapéutica integral, que integre la evidencia científica, la disponibilidad de las nuevas tecnologías, la costoefectividad y pertinencia de las intervenciones y la experiencia adquirida. En el ámbito de la medicina materno fetal, hacen falta más estudios y consensos en cuanto a protocolos de manejo. En muchas situaciones la conducta debe ser individualizada.

Es importante tener presente el manejo interdisciplinario de acuerdo a las condiciones institucionales y sobrevidas locales, puesto que sirve de apoyo para la toma de decisiones, no solo para la familia, que es pieza fundamental, sino también al médico tratante, haciendo énfasis en las consideraciones éticas y morales. Se debe enfatizar el principio básico de la medicina: "Primum non nocere" (Primero no hacer daño) porque no solo se busca preservar la vida de las personas sino evitar las menores consecuencias en estos y una buena calidad de vida.
En este caso se decidió el manejo intervencionista, toracocentesis más derivación pleuroamniótica, a pesar que la paciente se encontraba en la semana 32 de gestación, sin tener resultados de Test de Fisher, ni realización de estudio de anticuerpos irregulares y pruebas de maduración pulmonar. El feto no mostraba signos de anormalidades anatómicas o signos ecográficos de aneuploidias, que en algunos casos son evidentes como las trisomías, como el Síndrome de Down. Nos preguntamos: ¿En este paciente se justificarían dos intervenciones intrauterina? ¿Por qué no se consideró solo realizar toracocentesis e inmediatamente cesárea? ¿Por qué realizar nueva intervención y no interrumpir el embarazo, si la paciente estaba alrededor de las 33 semanas? ¿Hubiese sido conveniente el manejo extrauterino del feto, como lo demuestra la literaturas? Se insiste que el hidrotórax fetal es una situación difícil para la toma de decisiones. Debe ser manejado por un equipo interdisciplinario. Las elevadas tasas de mortalidad fetal conllevan la pregunta, si los recursos que se invierten podrían disponerse mejor para otras patologías de mejor pronóstico. Son importantes las implicaciones éticas que aporta el manejo expectante $o$ intervencionista en una entidad de elevada mortalidad.

No es fácil para el clínico ya que no solo debe tener en cuenta la evidencia científica y la parte locativa sino también el deseo de la paciente y la familia. A pesar que están demostradas las altas tasas de mortalidad, el pequeño porcentaje de sobrevida tiene un valor significativo para las familias. Lo que obliga a implementar todos los esfuerzos para preservar la vida de este nuevo ser.

Actualmente no existe un consenso del manejo óptimo del hidrotórax fetal aislado o asociado a hidropesía, cada día la ciencia sigue intentando resolver los problemas de salud. Se hace necesario que las terapéuticas médicas no estén basadas solo en reportes de casos, deben estar apoyadas en la mejor evidencia científica.

CONFLICTOS DE INTERÉS: ninguno que declarar. 
FINANCIACIÓN: recursos propios de los autores. Estudios de laboratorios, material quirúrgico, estudios paraclínicos, insumos hospitalarios y honorarios de los profesionales tratantes fueron aportados dentro de la atención asistencial.
AGRADECIMIENTOS: a la materna por su contribución desinteresada para nuestra estructuración conceptual. A los profesores Edgar Gómez, Antonio Fernández y Álvaro Olivera por la contribución y orientación dentro del proceso de conformación de este articulo.

\section{REFERENCIAS BIBLIOGRÁFICAS}

1. Flores X, Del Solar G, Sepulveda W, Hidrotórax fetal: Evaluación y manejo prenatal, Revista Chilena de Ultrasonografia, 2002; 5.

2. Cárdenas J, Huertas E, Ore I, Carvajal S, Hidrops fetal no Inmune: A propósito de un caso. Anales de la facultad de Medicina, Universidad Nacional Mayor de San Marcos, 2004; 65: 133137

3. Maya D, Leis MT, Hernández E, Karchmer SM. Hidropesía fetal no inmune: Revisión de la literatura y presentación de una propuesta para su estudio y manejo. Perinatol Reprod Hum $1995 ; 9: 131-141$.

4. Acho S, Díaz J, Guillen D, Bambarén E, Navarro R. Hidropesía fetal no inmunológica e infección intrauterina por citomegalovirus. Perinat Ginecol Obstet 2005;51:53-57.

5. Abrams M, Meredith K, Kinnard P, Clark R. Pediatrics of journal of the American Academy of Pediatrics, 2007;120

6. Borobio V, Guias clínicas medicina materno-fetal clínica barcelona, 2008.

7. Watson D, Campbell S. Antenatal Evaluation and management in nonimmune Hydrops Fetalis. Obstet Gynecol 1992; 79: 256-59.

8. Chavkin Y, Kupfersztain C, Ergaz L, Guedj P, Finkel A, Stark M. Successful Outcome of Idiopathic Nonimmune Hydrops Fetalis treated by Maternal difoxine. Ginecol Obstet Invest 1992; 742: 137-39.

9. Apkon M. Pathophysiology of hydrops fetalis. Semin perinatol 1995; 19: 437-46.

10. Trainor B, Tumban R. The emerging pattern of hidrops fetalis- incidence, aetiology and management. Ulster Med J 2006;75:185-186.

\section{GRUPO DE INVESTIGACIÓN: MICROBIOLOGÍA CLÍNICA AMBIENTAL}

LÍNEAS: Enfermedad Diarreica Aguda (EDA). Enfermedades de Transmisión Sexual (ETS). Infecciones Intrahospitalarias. Microbiología Ambiental Email: sarzuza33@yahoo.es 